02,05

\title{
Модель поведения гранулярного ВТСП во внешнем магнитном поле: температурная эволюция гистерезиса магнитосопротивления
}

\author{
(C) С.В. Семенов ${ }^{1,2}$, Д.А. Балаев ${ }^{1,2}$ \\ ${ }^{1}$ Институт фризики им. Л.В. Киренского ФИЦ КНЦ СО РАН, \\ Красноярск, Россия \\ ${ }^{2}$ Сибирский федееральный университет, \\ Красноярск, Россия \\ E-mail: svsemenov@iph.krasn.ru
}

Поступила в Редакцию 13 февраля 2020 г.

В окончательной редакции 13 февраля 2020 г.

Принята к публикации 18 февраля 2020 г.

Модель, описывающая поведение магнитосопротивления $R(H)$ гранулярного высокотемпературного сверхпроводника (ВТСП), развиваемая в последнее десятилетие, дает объяснение достаточно необычному виду и таким особенностям гистерезисных зависимостей $R(H)$ (при $T=\mathrm{const}$ ), как локальный максимум, участок с отрицательным магнитосопротивлением, локальный минимум, и др. В рамках этой модели рассматривается эффективное поле в межгранульной среде $\mathbf{B}_{\text {eff, }}$ которое является суперпозицией внешнего поля и поля, индуцированного магнитными моментами ВТСП гранул. Оно может быть записано в виде: $\mathbf{B}_{\text {eff }}(H)=\mathbf{H}+4 \pi \alpha \mathbf{M}(H)$, где $M(H)$ - экспериментальная зависимость намагниченности, $\alpha$ - параметр, характеризующий сгущение линий магнитной индукции в межгранульной среде. В результате магнитосопротивление является не просто функцией внешнего поля, но и „внутреннего“, эффективного поля: $R(H)=f\left(\mathbf{B}_{\text {eff }}(H)\right)$. Исследовано магнитосопротивление гранулярного ВТСП $\mathrm{YBa}_{2} \mathrm{Cu}_{3} \mathrm{O}_{7-\delta}$ в широком диапазоне температур. Экспериментальные гистерезисные зависимости $R(H)$, полученные в диапазоне высоких температур (77-90K), хорошо объясняются в рамках этой модели, и значение параметра $\alpha$ составляет 20-25. Однако для температуры $4.2 \mathrm{~K}$ локальные экстремумы не наблюдаются, хотя выражение для $\mathbf{B}_{\text {eff }}(H)$ предсказывает их наличие, а параметр $\alpha$ несколько вырастает $(\sim 30-35)$ для этой температуры. Дополнительным фактором, который необходимо учитывать в этой модели, может быть перераспределение траекторий микроскопического тока, также влияющее на процессы диссипации в межгранульной среде. Для области низких температур и в условиях сильного сжатия магнитного потока $(\alpha \sim 30-35)$ возможно изменение микроскопических траекторий тока $\mathbf{I}_{m}$ при котором предпочтительнее туннелирование через соседнюю гранулу, но угол между $\mathbf{I}_{m}$ и $\mathbf{B}_{\text {eff }}$ будет заметно меньше $90^{\circ}$, хотя направления внешнего поля (а также эффективного поля) и макроскопического тока взаимно перпендикулярны.

Ключевые слова: гранулярные ВТСП, гистерезис магнитосопротивления, межгранульные границы.

DOI: 10.21883/FTT.2020.07.49464.029

\section{1. Введение}

Поликристаллические (далее - гранулярные) высокотемпературные сверхпроводники (ВТСП) представляют собой двухуровневую сверхпроводящую систему. Это - сверхпроводящие кристаллиты („сильная“ сверхпроводящая подсистема), связанные посредством эффекта Джозефсона через межгранульные границы, которые образуют уже вторую, „слабую“ сверхпроводящую подсистему. Вклад межгранульных границ в магнитные свойства проявляется только в достаточно слабых магнитных полях: порядка десятков Эрстед в низких температурах $[1,2]$ и долей Эрстеда в высоких температурах [2,3]. В умеренных и сильных магнитных полях намагниченность гранулярных ВТСП определяется только откликом гранул $[4,5]$, фактически, внутригранульным критическим током $[5,6]$. Подсистема межгранульных границ, в свою очередь, определяет транспортные свойства гранулярных ВТСП, поскольку перенос сверхпроводящего тока через объемный обра- зец происходит путем туннелирования носителей через границы между гранулами [7-9].

Однако есть и взаимодействие указанных подсистем. В наибольшей степени оно проявляется в магнитотранспортных свойствах - зависимостях критического тока от магнитного поля [10-15] и магнитосопротивлении $R(H)$ [13-16]. Например, зависимости $R(H)$ проявляют сложный немонотонный и гистерезисный характер [17-20], который не был объяснен в ранних работах. Поэтому модель поведения гранулярного ВТСП во внешнем магнитном поле начала формироваться только в 2000 годы [21-44], когда стали появляться работы, посвященные детальному исследованию магнитотранспортных свойств.

Вкратце, взаимодействие подсистем гранул и границ может быть объяснено следующим образом. Линии магнитной индукции от магнитных моментов MG BTCП гранул замыкаются через межгранульное пространство, см. рис. 1, $a$. В результате поле в межгранульных промежутках отличается от внешнего поля, и именно оно 
влияет на процессы туннелирования между гранулами. Индуцированное магнитными моментами $\mathbf{M}_{\mathrm{G}}$ поле $\mathbf{B}_{\text {ind }}$ пропорционально удельной намагниченности $M$ (фактически - усредненному магнитному моменту гранул), и оно будет гистерезисной функцией внешнего поля $H$ ввиду известного для сверхпроводников II рода гистерезиса $M(H)$. Следовательно, эффективное поле $\mathbf{B}_{\text {eff }}$ в межгранульной среде можно записать в виде

$$
\mathbf{B}_{\text {eff }}(H) \sim \mathbf{H}+\mathbf{B}_{\text {ind }}(H) .
$$

Выражение (1) дает объяснение гистерезисному характеру зависимостей $R(H)$ поскольку диссипация в межгранульных границах определяется эффективным

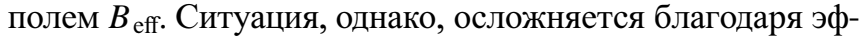
фекту сжатия магнитного потока. Дело в том, протяженность межгранульных границ имеет величину порядка сверхпроводящей длины когерентности (единицы $\mathrm{nm}$ ), в то время как размеры гранул несоизмеримо больше (единицы - десятки $\mu \mathrm{m}$ ). Такое различие в размерах неизбежно приводит к сильному сжатию линий магнитной индукции в межгранульной среде, схематически показанному на рис. $1, b$. На возможность реализации эффекта сжатия потока в межгранульной среде было впервые указано в работе [21], и впоследствии это было экспериментально подтверждено в ряде экспериментов [45-51]. В частности, было предложено ввести коэффициент пропорциональности между $B_{\text {ind }}$ и намагниченностью $M: B_{\text {ind }}=4 \pi \alpha M$. Здесь параметр $\alpha$ характеризует усредненный эффект сгущения линий магнитной индукции $B_{\text {ind. }}$. Тогда выражение $(1)$ переписывается в виде:

$$
B_{\text {eff }}(H)=|H-4 \pi \alpha M(H)| .
$$

В выражении (2) учтено направление линий магнитной индукции $B_{\text {ind }}$ относительно внешнего поля $H$ (см. рис. $1, a, b)$. Модуль взят ввиду того, что сопротивление - четная функция магнитного поля (множитель $4 \pi$ соответствует системе СГС).

Магнитосопротивление гранулярного сверхпроводника $R(H)$ является функцией $B_{\text {eff. }}$ И если рассматривать процессы диссипации в рамках стандартных подходов, например, аррениусовского соотношения $R(H)=R_{\mathrm{NJ}} \exp (-U / k T)$, то необходимо понимать, что энергия джозефсоновской связи $U$ (эквивалент потенциала пиннинига вихрей Абрикосова для ВТСП-гранул) в первую очередь является функцией эффективного поля, а не внешнего поля $\left(U(H) \rightarrow U\left(B_{\text {eff }}\right)\right)$. Описываемый подход хорошо воспроизводит основные особенности наблюдаемых зависимостей $R(H)$ с помощью выражения (2) и экспериментальных петель гистерезиса намагниченности $M(H)$ [52]. Детальное сопоставление гистерезисных зависимостей $R(H)$ с зависимостями $B_{\text {eff }}(H)$ показало, что величина параметра $\alpha$ для ВТСП-структуры 1-2-3 составляет $\approx 20-25$, и степень сжатия практически не меняется от $77 \mathrm{~K}$ до температуры сверхпроводящего перехода $(\approx 90 \mathrm{~K})$ [50]. Для дальнейшего развития модели поведения гранулярного ВТСП во
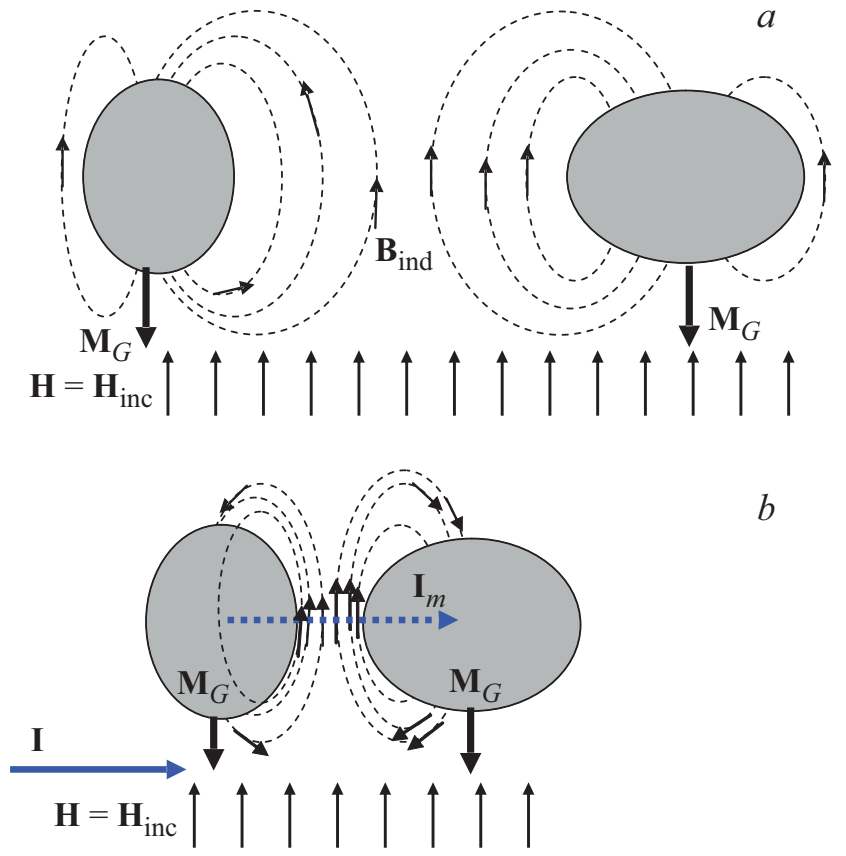

$b$

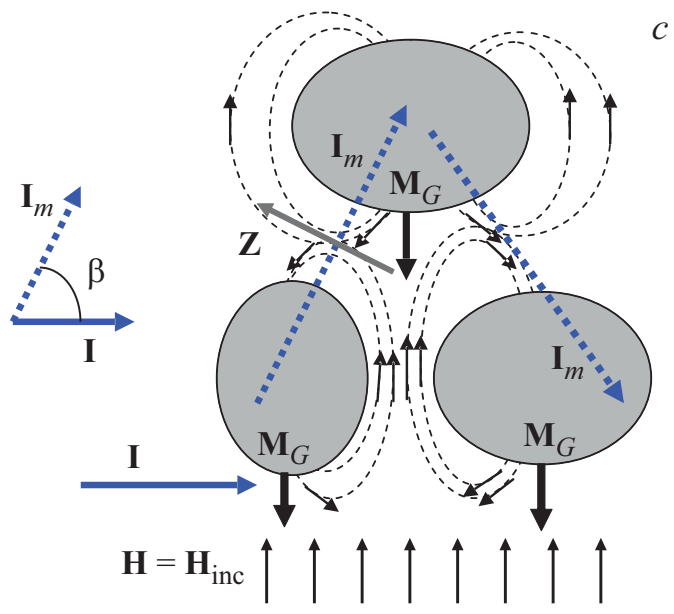

Pис. 1. Схематическое представление линий магнитной индукции в межгранульной среде гранулярного ВТСП. Овалы ВТСП-гранулы, пространство между ними - межгранульная среда; в данном представлении межгранульные промежутки значительно увеличены. Пунктирные линии - линии магнитной индукции $\mathbf{B}_{\text {ind }}$ от магнитных моментов сверхпроводящих гранул $\mathbf{M}_{\mathrm{G}}$, стрелки показывают направление $\mathbf{B}_{\text {ind }}$ при возрастающем внешнем поле $\mathbf{H}=\mathbf{H}_{\text {inc. }}$. На частях $(a)$ и $(b)$ в сравнении показаны случаи, когда гранулы находятся, соответственно, ,далеко“ и „близко“ друг от друга. В последнем случае $b$ реализуется эффект сгущения линий магнитной индукции. На $b$ и $c \mathbf{I}_{\mathrm{m}}-$ микроскопические траектории тока, $\mathbf{I}-$ направление макроскопического тока при „перпендикулярной“ ориентации $\mathbf{H} \perp \mathbf{I}$. На $c$ показано возможное перераспределение траекторий $\mathbf{I}_{\mathrm{m}}$, если угол между $\mathbf{I}_{\mathrm{m}}$ и $\mathbf{B}_{\text {ind }}$ мал (см. разд. 3).

внешнем магнитном поле необходимо более существенное расширение температурного диапазона исследований. В настоящей работе проведены измерения гистерезисных зависимостей $R(H)$ образца гранулярного ВТСП 
$\mathrm{YBa}_{2} \mathrm{Cu}_{3} \mathrm{O}_{7}$ как в области высоких температур, так и при температуре жидкого гелия. Основной целью данных исследований было проследить изменение формы зависимости $R(H)$ с температурой для проверки, дополнения и дальнейшего развития модели гранулярного ВТСП.

\section{2. Экспермент}

Образец ВТСП $\mathrm{YBa}_{2} \mathrm{Cu}_{3} \mathrm{O}_{7-\delta}$ был приготовлен стандартным методом твердофазного синтеза из соответствующих оксидов с тремя промежуточными помолами; окончательная стадия отжига проводилась при температуре около $940^{\circ} \mathrm{C}$ (близкой к температуре плавления) в течении $50 \mathrm{~h}$. По окончании синтеза образец был отожжен при температуре $350^{\circ} \mathrm{C}$ в течение $10 \mathrm{~h}$ для достижения стехиометрии по кислороду.

Согласно результатам рентгеноструктурного анализа, все рефлексы полученного образца соответствуют ВТСП со структурой 1-2-3, посторонних фаз замечено не было. По данным сканирующей электронной микроскопии (использовался электронный микроскоп Hitachi-TM 3000) средний размер гранул $d$ составил около $\sim 10 \mu \mathrm{m}$, причем, есть области, в которых происходит срастание кристаллитов. Соотношение элементов, согласно результатам энергодисперсионной спектрометрии, соответствует химической формуле $\mathrm{YBa}_{2} \mathrm{Cu}_{3} \mathrm{O}_{7-\delta}$. Температура сверхпроводящего перехода $T_{C}$, определенная из магнитных измерений (см. рис. 2) составила $92.4 \mathrm{~K}$.

Измерения транспортных свойств проводились стандартным четырехзондовым методом. Величина плотности критического тока $J_{C}$ составила $\approx 150 \mathrm{~A} / \mathrm{cm}^{2}$ при температуре $T=77 \mathrm{~K}$ и $\approx 1.5 \mathrm{kA} / \mathrm{cm}^{2}$ при $T=4.2 \mathrm{~K}$ (в отсутствии внешнего поля). Для образцов с такими, достаточно высокими значениями $J_{C}$, есть экспериментальная проблема при проведении транспортных измерений. Необходимо проводить измерения в транспортном токе $I$ порядка критического $I_{C}$. Если $I<I_{C}$ в некотором поле $H$, то $R(H)=0$, следовательно, для измерения зависимостей $R(H)$ необходимо выполнение условия $I>I_{C}(H)$. При типичных размерах образца $\approx 0.8 \times 0.8 \times 8 \mathrm{~mm}^{3}$ (транспортный ток $I$ прикладывается вдоль длинного направления) при $T=4.2 \mathrm{~K}$ необходимо, чтобы транспортный ток $I$ достигал значений более $\sim 200 \mathrm{~mA}$, и чтобы обеспечить эффективный отвод тепла, выделяющегося на контактах, необходимо, чтобы образец находился в криогенной жидкости. В настоящей работе использовались прижимные позолоченные электрические контакты. Такие контакты позволили избежать разогрева образца из-за выделения тепла на токовых контактах при транспортных токах до $30 \mathrm{~mA}$, если образец находился в гелиевой теплообменной атмосфере, и, по крайней мере, до $500 \mathrm{~mA}$, при помещении образца непосредственно в криогенную жидкость. Внешнее поле, задаваемое либо электромагнитом (высокие температуры, $I=30 \mathrm{~mA}$ ), либо сверхпроводящим соленоидом, прикладывалось перпендикулярно направлению транспортного тока $(\mathbf{H} \perp \mathbf{I})$. Измерения $R(H)$ при малом

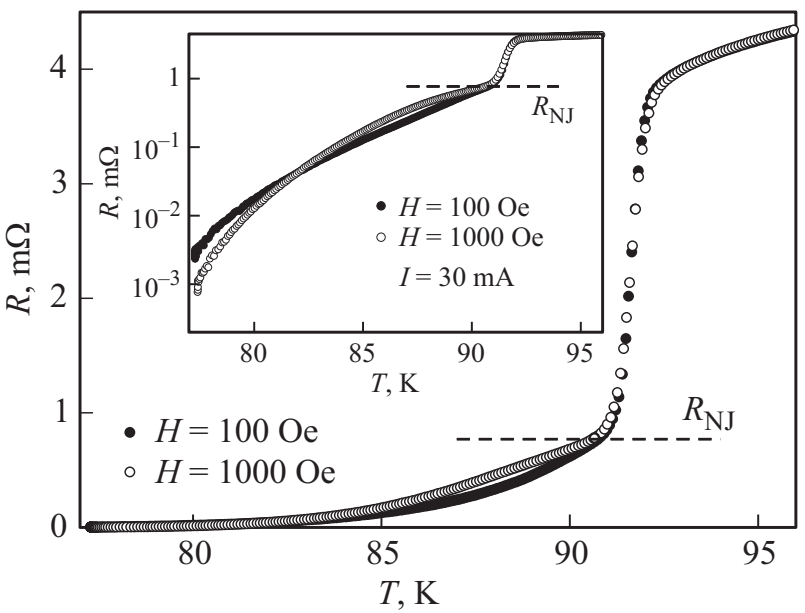

Рис. 2. Температурные зависимости электросопротивления $R(T)$ исследованного образца во внешних магнитных полях 100 и $1 \mathrm{kOe}$. На вставке - то же самое в полулогарифмических координатах. Горизонтальная штриховая линия соответствует полному сопротивлению подсистемы межгранульных границ $-R_{\mathrm{NJ}}$.

( $I=1 \mathrm{~mA})$ значении транспортного тока была проведены с использованием установки PPMS-6000. Данные по магнитосопротивлению как при высоких температурах, так и $4.2 \mathrm{~K}$ (в криостате с жидким гелием), были получены на одном и том же образце.

Магнитные свойства исследованы на вибрационном магнетометре [53] при внешних условиях, соответствующих магнитотранспортным измерениям, на том же самом образце, на котором измерялись зависимости $R(H)$.

\section{3. Результаты и обсуждение}

На рис. 2 приведены температурные зависимости электросопротивления $R(T)$ во внешних магнитных полях $100 \mathrm{Oе}$ и $1 \mathrm{kOe}$. Резкий скачок сопротивления, начинающийся при $T_{C} \approx 92.4 \mathrm{~K}$, слабо зависящий от внешнего поля, соответствует переходу в подсистеме сверхпроводящих гранул, а плавная часть зависимостей $R(T)$ отражает переход в сверхпроводящее состояние подсистемы межгранульных границ $[7,15,19,20,31,32,39]$. Такое четкое разграничение диссипации в подсистемах гранул и межгранульных границ отражает справедливость рассмотрения гранулярного ВТСП, как двухуровневой подсистемы (см. разд. 1). Если условно разделить полное сопротивление образца как сумму сопротивлений гранул и межгранульных границ, то значение $R$ в близости начала перехода в межгранульных границах можно считать „нормальным“ сопротивлением $R_{\mathrm{NJ}}$ этой подсистемы [54] (см. рис. 2).

Обычно для сверхпроводников II рода зависимости $R(T)$ во внешнем поле являются монотонными функциями, и при $T=$ const сопротивление тем больше, чем больше внешнее поле [55]. Несколько иная ситуация 

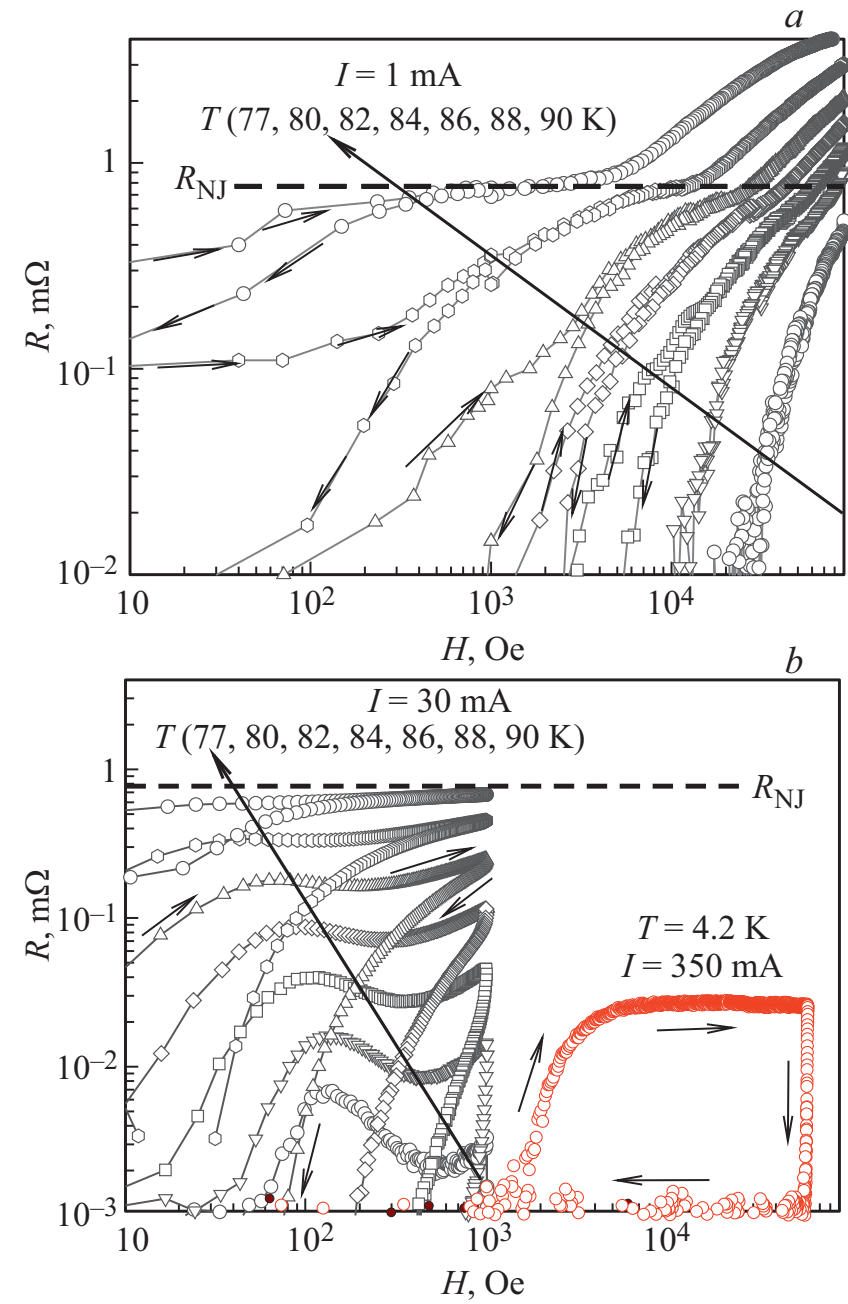

Рис. 3. Гистерезисные зависимости $R(H)$ при указанных температурах и значениях транспортного тока $I$ в двойной логарифмической шкале. Стрелки показывают направление изменения внешнего поля. Величина $R_{\mathrm{NJ}}$ (см. рис. 2) показана горизонтальной штриховой линией.

наблюдается для данных рис. 1. Из вставки на рис. 1 видно, что зависимости $R(T)$ во внешних полях $100 \mathrm{Oe}$ и $1 \mathrm{kOe}$ пересекаются, и в температурном диапазоне $77-82 \mathrm{~K} R(H=1 \mathrm{kOe})<R(H=100 \mathrm{Oe})$, в то время как при $T>83 \mathrm{~K}$ имеет место стандартное поведение: $R(H=100 \mathrm{Oe})<R(H=1 \mathrm{kOe})$. Такая необычная особенность будет объяснена ниже, на основании анализа гистерезисных зависимостей $R(H)$.

Температурная эволюция зависимости $R(H)$ показана на рис. 3. Рис. $3, a$ содержит информацию о поведении $R(H)$ при малом значении транспортного тока $(I=1 \mathrm{~mA})$ до полей $90 \mathrm{kOe}$ при температурах от 77 до $90 \mathrm{~K}$. Зависимости $R(H)$, измеренные при значительно большем токе $I=30 \mathrm{~mA}$ в полях до $1 \mathrm{kOe}$ и в том же температурном диапазоне (77-90 K), приведены на pис. $3, b$. Также на рис. $3, b$ показана гистерезисная зависимость магнитосопротивления при температуре $4.2 \mathrm{~K}$; она была получена при транспортном токе $I=350 \mathrm{~mA}$.
На рис. 3 использована двойная логарифмическая шкала ввиду большого диапазона сопротивления и магнитных полей. Горизонтальные линии на рис. 3 показывают величину „нормального“ сопротивления подсистемы межгранульных границ $-R_{\mathrm{NJ}}$. Как видно из рис. $3, a$, в достаточно большом внешнем поле сопротивление образца достигает этой величины. В поле $H^{*}$, в котором $R \approx R_{\mathrm{NJ}}$ зависимости $R(H)$ демонстрируют особенность - смену знака кривизны. Это свидетельствует о выходе на насыщение (плато) магнитосопротивления подсистемы межгранульных границ $[38,39]$. С дальнейшим увеличением поля сопротивление образца превышает величину $R_{\mathrm{NJ}}$, и при $H \geq H^{*}$ начинается диссипация уже в сверхпроводящих гранулах. Гистерезис магнитосопротивления существует в диапазоне полей от нуля до $H^{*}$.

Гистерезисное поведение магнитосопротивления качественно имеет одинаковый характер как для „малого“ транспортного тока $(1 \mathrm{~mA}-$ рис. $3, a)$, так и для $I=30 \mathrm{~mA}$ и более (рис. $3, b): R\left(H_{\text {inc }}\right)$ практически всегда больше $R\left(H_{\mathrm{dec}}\right)$; здесь и далее $H_{\mathrm{dec}}$ и $H_{\mathrm{inc}}-$ соответствуют убывающему и возрастающему внешнему полю. Зависимости $R\left(H_{\text {inc }}\right)$ на рис. $3, b$ демонстрируют немонотонное поведение - на них присутствуют явно выраженные максимум и минимум при возрастании поля. Из температурной эволюции зависимостей $R(H)$ на рис. $4, b$ видно, что при $T=77$ и $80 \mathrm{~K}$ выполняется неравенство $R(H \approx 100 \mathrm{Oe})>R(H \approx 1 \mathrm{kOe})$, тогда как при $T=82 \mathrm{~K}$ сопротивления в этих полях примерно равны, а в области температур, больших $82 \mathrm{~K}, R\left(H_{\text {inc }} \approx 100 \mathrm{Oe}\right)$ уже меньше $R\left(H_{\mathrm{inc}} \approx 1 \mathrm{kOe}\right)$. Это коррелирует с „нетипичным“ поведением зависимостей $R(T)$, измеренных при этом же значении тока $(I=30 \mathrm{~mA})$ (см. вставку на рис. 2).

Сравнивая данные рис. $3, a$ и $b$ для диапазона температур 77-90 K, можно сказать, что в малом транспортном токе характерные локальные экстремумы зависимостей $R\left(H_{\text {inc }}\right)$ не видны. Это происходит из-за того, что в случае достаточно малого транспортного тока диссипация начинается в полях, больших, чем поле, в котором появляются аномалии зависимостей $R\left(H_{\text {inc }}\right)$. Зависимость $R(H)$ при $T=4.2 \mathrm{~K}$, показанная на pис. $3, b$, была получена еще в большем транспортном токе $(I=350 \mathrm{~mA})$, чем данные для температур 77-90 K на этом же рисунке. Видно, что зависимость $R\left(H_{\text {inc }}\right)$ при $T=4.2 \mathrm{~K}$ уже не содержит характерных локальных экстремумов, что, как будет показано ниже, является нетривиальным фактом. Далее рассмотрим более детально влияние транспортного тока на вид гистерезиса магнитосопротивления, а также происхождение локальных экстремумов зависимости $R\left(H_{\mathrm{inc}}\right)$.

На рис. 4 приведены зависимости $R(H)$ при различных величинах тока $I$ для температур 77 и $4.2 \mathrm{~K}$ в полях до 1 и $10 \mathrm{kOe}$ соответственно (использована двойная логарифмическая шкала). Для данных при $T=77 \mathrm{~K} \mathrm{c}$ увеличением тока магнитосопротивление возрастает, и несколько видоизменяется форма зависимостей $R(H)$. 


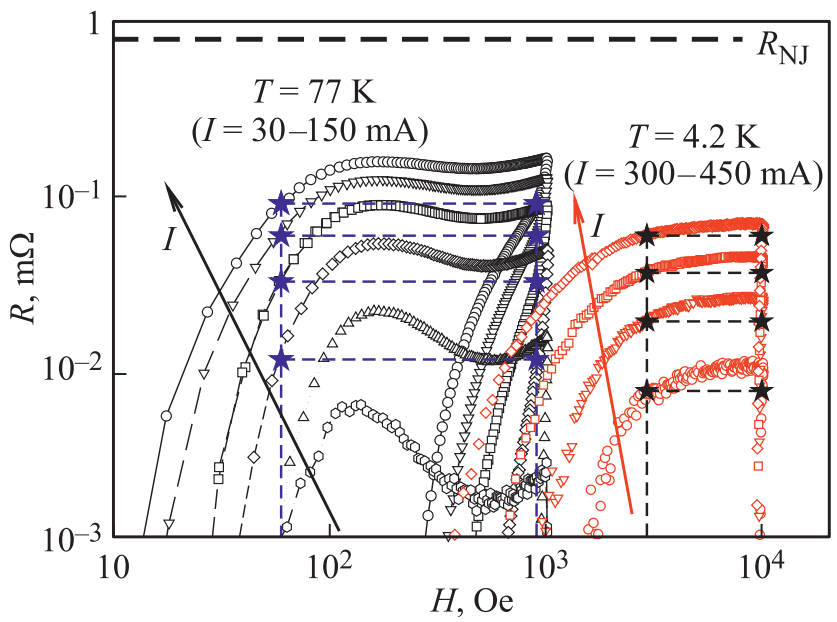

Рис. 4. Зависимости $R(H)$ при $T=77 \mathrm{~K}(I=30,50,75,100$, $125,150 \mathrm{~mA})$ и $4.2 \mathrm{~K}(I=300,350,400,450 \mathrm{~mA})$ в двойной логарифмической шкале. Горизонтальные и вертикальные штриховые линии показывают одинаковую величину ширины гистерезиса магнитосопротивления для различных значений $I$.

Максимум и минимум зависимости $R\left(H_{\text {inc }}\right)$ присутствуют для всех использованных величин транспортного тока, и их положение не сильно меняется для различных значений $I$. При $T=4.2 \mathrm{~K}$ увеличение тока от 300 до $450 \mathrm{~mA}$ приводит только к тому, что заметное магнитосопротивление появляется в меньших полях $H_{\text {inc }}$.

Ранее было показано, что в диапазоне достаточно высоких температур (от $77 \mathrm{~K}$ до $T_{C}$ ) гистерезисные зависимости $R(H)$ гранулярных ВТСП проявляют универсальное поведение: при $T=$ const полевая ширина гистерезиса не зависит от транспортного тока $[34,35,54,47,50]$. Этот параметр, фактически, является длиной отрезка, соединяющего точки $H_{\mathrm{dec}}$ и $H_{\mathrm{inc}}$ гистерезисной зависимости $R(H)$, при условии $R\left(H_{\mathrm{dec}}\right)=R\left(H_{\mathrm{inc}}\right)$ :

$$
\Delta H=H_{\mathrm{dec}}-H_{\mathrm{inc}} .
$$

Такое поведение параметра $\Delta H$ является прямым следствием реализации в гранулярном ВТСП двухуровневой сверхпроводящей системы, в которой транспортный ток влияет на диссипацию в подсистеме межгранульных границ, но не может повлиять на намагниченность гранул и изменить поле $B_{\text {ind. }}$ Горизонтальные линии, соединяющие точки на рис. 4 , соответствуют полевой ширине гистерезиса магнитосопротивления $\Delta H=H_{\mathrm{dec}}-H_{\text {inc }}$ при $H_{\mathrm{dec}}=900 \mathrm{Oе}$ для данных при $77 \mathrm{~K}$ и $H_{\mathrm{dec}}=9800 \mathrm{Oe}$ для данных при $4.2 \mathrm{~K}$. Точки пересечения горизонтальных линий с зависимостью $R\left(H_{\text {inc }}\right)$ имеют одинаковые абсциссы (показано вертикальными штриховыми линиями). Это иллюстрирует независимость полевой ширины гистерезиса от транспортного тока (для других значений $H_{\text {dec }} \Delta H$ также не зависит от $\left.I\right)$. Ранее для гранулярных ВТСП иттриевой системы это свойство было описано только для температуры $77 \mathrm{~K}[47,50,54]$, и в настоя- щей работе получено экспериментальное подтверждение независимости $\Delta H$ от транспортного тока при $T=4.2 \mathrm{~K}$.

Рассмотрим происхождение локальных экстремумов зависимости $R\left(H_{\text {inc }}\right)$. На рис. 5 приведены гистерезисные зависимости намагниченности $M(H)$, магнитосопротивления $R(H)$, эффективного поля $B_{\text {eff }}(H)$ при $T=80 \mathrm{~K}$.
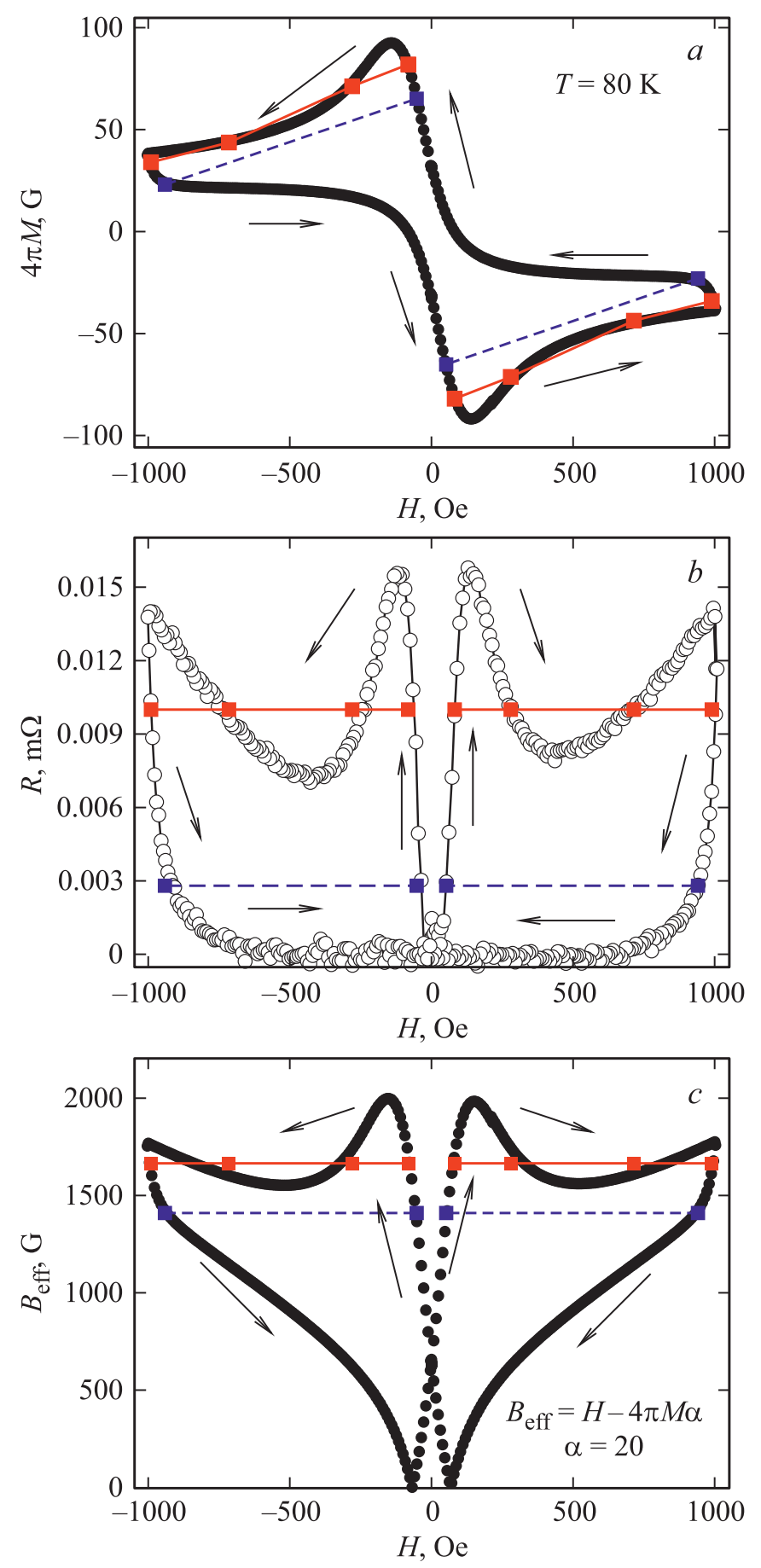

Рис. 5. Гистерезисные зависимости: $a-M(H), b-R(H)$, $c-\mathbf{B}_{\mathrm{eff}}(H)$ при $T=80 \mathrm{~K}$. Горизонтальные линии на $b$ и $c$ иллюстрируют, то, что значения $\Delta H$ между точками на зависимостях $R(H)$ и $\mathbf{B}_{\text {eff }}(H)$ примерно одинаковы. Стрелки показывают направление изменения внешнего поля. 

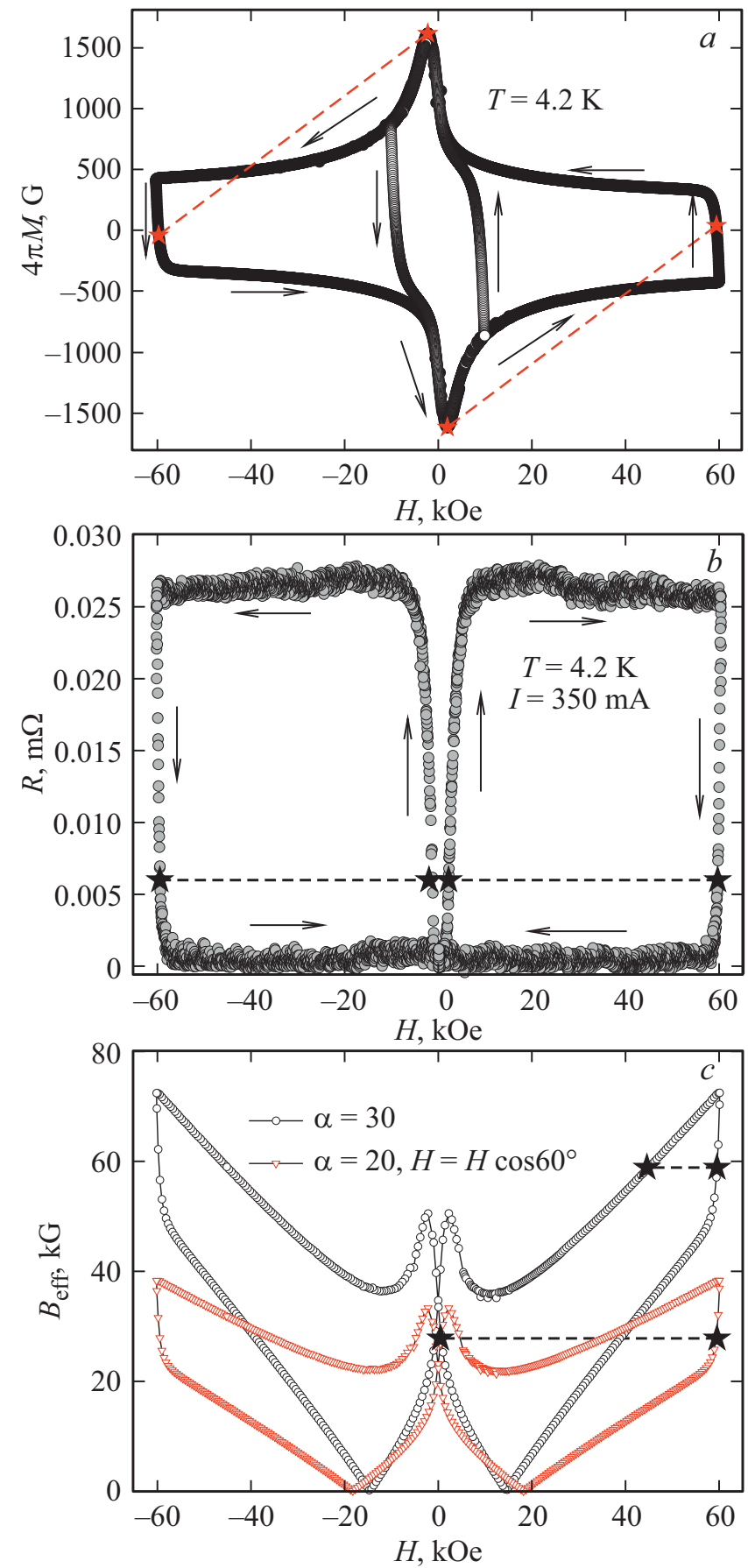

Рис. 6. Гистерезисные зависимости: $a-M(H), b-R(H)$, $c-\mathbf{B}_{\text {eff }}(H)$ при $T=4.2 \mathrm{~K}$. Горизонтальные линии на $b$ и $c$ иллюстрируют ширину гистерезиса $R(H)$ и $\mathbf{B}_{\text {eff }}(H)$ при $H_{\mathrm{dec}}=59.4 \mathrm{kOe}$. $\mathbf{B}_{\mathrm{eff}}(H)$ на $c$ построены по выражению (2) при параметрах, указанных на рисунке.

Выражение (2) для эффективного поля в межгранульной среде содержит неизвестный параметр $\alpha$. Его значение можно определить исходя из следующих соображений. Для любой точки зависимости $R\left(H_{\mathrm{dec}}\right)$ есть точка на зависимости $R\left(H_{\mathrm{inc}}\right)$, в которой $R\left(H_{\mathrm{dec}}\right)=R\left(H_{\mathrm{inc}}\right)$. Тогда и эффективное поле $B_{\text {eff }}$ в точках с этими абсциссами
$\left(H_{\mathrm{dec}}\right.$ и $\left.H_{\mathrm{inc}}\right)$ будет одинаковым: $B_{\mathrm{eff}}\left(H_{\mathrm{dec}}\right)=B_{\mathrm{eff}}\left(H_{\mathrm{inc}}\right)$. Ширина гистерезиса $R(H)$, определяемая выражением (3), не зависит от транспортного тока, а значит, она должна иметь то же значение, что и $\Delta H$ для эффективного поля: $\Delta H_{R=\text { const }}=\Delta H_{B_{\text {eff }}=\text { const. }}$ Следовательно, при значении параметра $\alpha$, дающем наилучшее согласие между величинами $\Delta H$ для гистерезиса $R(H)$ и гистерезиса $B_{\text {eff }}(H)$, полученная зависимость $B_{\text {eff }}(H)$ будет адекватно отражать эффективное поле в межгранульной среде. Зависимость $B_{\text {eff }}(H)$ на рис. 5, $c$ построена при $\alpha=20$ с использованием экспериментальных данных по намагниченности рис. 5, $a$. Как видно из рис. $5, b$ и $c$, длины горизонтальных отрезков (это и есть ширина гистерезиса $\Delta H)$, пересекающих зависимости $R(H)$ и $B_{\text {eff }}(H)$, практически одинаковы. Согласие длин отрезков имеет место как в случае, когда горизонтальные прямые $R=$ const $\left(\right.$ или $B_{\text {eff }}=$ const) четыре раза пересекают зависимости $R(H)$ и $B_{\text {eff }}(H)$, так и когда прямые $R=\mathrm{const}$ только два раза пересекают их (нижние прямые на рис. $3, b, c)$. На зависимости $M(H)$ (рис. 5,a) также показаны точки, соответствующие условиям $R=\mathrm{const}$ и $B_{\text {eff }}=$ const на рис. $5, b$ и $c$ соответственно. Сравнивая данные на рис. $5, a, b$ и $c$, можно однозначно заключить, что локальный максимум зависимости $R\left(H_{\text {inc }}\right)$ соответствует экстремуму зависимости $M\left(H_{\text {inc }}\right)$. $\mathrm{C}$ понижением температуры от 88 до $77 \mathrm{~K}$ положение максимума зависимости $R\left(H_{\text {inc }}\right)$ смещается в область больших полей (см. рис. $3, b)$. Это вызвано смещением положения экстремума зависимости $M\left(H_{\text {inc }}\right)$ с понижением температуры.

Рассмотрим подробнее магнитосопротивление при $T=4.2 \mathrm{~K}$. Зависимость $R(H)$ в диапазоне полей $\pm 60 \mathrm{kOe}(I=350 \mathrm{~mA})$ показана на рис. $6, b$. Эта зависимость имеет вид „прямоугольной петли“, что кардинально отличается от данных для высоких температур (см. рис. $3, b, 5, b$ ). Сначала оценим значение параметра $\alpha$, характеризующего степень сжатия магнитного потока в межгранульной среде. Горизонтальные штриховые линии, соединяющие точки на рис. $6, b$, имеют тот же смысл, что и для рис. 4 и $5, b$, и на рис. $6, b$ эти линии соответствуют ширине гистерезиса магнитосопротивления $\Delta H$ при $H_{\mathrm{dec}}= \pm 59.4 \mathrm{kOe}$. Абсцисса точки пересечения горизонтальных линий на рис. $6, b$ с зависимостью $R\left(H_{\text {inc }}\right)$ составляет $\pm 2.1 \mathrm{kOe}$ следовательно $\Delta H=57.3 \mathrm{kOe}$. Величины намагниченности образца в полях $H_{\mathrm{dec}}= \pm 59.4 \mathrm{kOe}$ и $H_{\text {inc }}= \pm 2.1 \mathrm{kOe}$ видны на рис. 6, $a$. Из выражений (2) и (3) при $B_{\text {eff }}\left(H_{\text {dec }}\right)=B_{\text {eff }}\left(H_{\text {inc }}\right)$, получим

$$
\Delta H=H_{\mathrm{dec}}-H_{\mathrm{inc}}=4 \pi \alpha\left\{M\left(H_{\mathrm{dec}}\right)-M\left(H_{\mathrm{inc}}\right)\right\} .
$$

Подставляя экспериментальные значения намагниченности $M\left(H_{\mathrm{dec}}\right), M\left(H_{\mathrm{inc}}\right)$ и $\Delta H=57.3 \mathrm{kOe}$ в выражение $(4)$, получим, что величина параметра $\alpha$ составляет около 30 . Следовательно, степень сжатия магнитного потока не уменьшилась при низкой температуре (при высоких температурах $\alpha \approx 20$ ), а скорее увеличилась. 
Однако в рамках вышеописанного подхода „прямоугольную“ форму зависимости $R(H)$ при $4.2 \mathrm{~K}$ на pис. $6, b$ объяснить проблематично. Действительно, зависимость $B_{\text {eff }}(H)$ на рис. $6, c$, построенная при $\alpha=30$ с использованием данных по намагниченности при $T=4.2 \mathrm{~K}$, обладает и локальным максимумом, и локальным минимумом, характерными и для высоких температур. Следует отметить, что для бо́льших значений $\alpha$ согласие между шириной гистерезиса $R(H)$ и $B_{\text {eff }}(H)$ не улучшается. Если же при построении $B_{\text {eff }}(H)$ уменьшать $\alpha$, то локальный максимум на зависимости $B_{\text {eff }}\left(H_{\text {inc }}\right)$ будет менее выражен, однако ввиду доминирования первого слагаемого в выражении (2) значение $\Delta H$ при $H_{\mathrm{dec}}= \pm 59.4 \mathrm{kOe}$ будет гораздо меньше величины $\Delta H$ зависимости $R(H)$.

Итак, из сравнительного анализа экспериментальных гистерезисных зависимостей $R(H)$ при высоких температурах и низкой температуре $(4.2 \mathrm{~K})$ и можно констатировать, что для низкой температуры модель гистерезисного поведения гранулярного ВТСП во внешнем поле дает только качественное согласие с экспериментом. И основным непонятным фактом является отсутствие локальных экстремумов зависимости $R\left(H_{\text {inc }}\right)$ при $T=4.2 \mathrm{~K}$ („прямоугольная“ форма). Рассмотрим возможные причины такого поведения. Хотя сопротивление образца при $T=4.2 \mathrm{~K}$, транспортных токах $I=300-450 \mathrm{~mA}$ и в полях $H \sim 10-60 \mathrm{kOe}$ составляет $\sim 3-4 \%$ от значения $R_{\mathrm{NJ}}$, тем не менее оно близко́ к сопротивлению при температурах $77-80 \mathrm{~K}$, транспортных токах $I=30-75 \mathrm{~mA}$ и в полях $H \sim 0.1-1 \mathrm{kOe}(\mathrm{cm}$. pис. $3, b$, и рис. 4). Следовательно, нельзя считать, что экспериментальные условия при $T=4.2 \mathrm{~K}$ аналогичны условиям „малого тока“, как для данных рис. $3, a$, когда наблюдаемая диссипация начинается в больших полях. Зависимости $R(H)$ при $T=4.2 \mathrm{~K}$, так же как и в области высоких температур, характеризуются отсутствием зависимости полевой ширины гистерезиса от транспортного тока, что дает основания для использования параметра $\Delta H$ для анализа и сравнения шириной гистерезиса эффективного поля $-B_{\text {eff }}(H)$. Степень сжатия потока в межгранульной среде, определяемая величиной $\alpha$, в низких температурах осталась примерно на том же уровне, что и в области высоких температур ( $\alpha \sim 20-30)$.

По-видимому, с понижением температуры появляется дополнительный фактор, влияющий на характер прохождения (туннелирования) носителей сверхпроводящего тока через межгранульные границы. Эти фактором может быть перераспределение траекторий микроскопических токов. Согласно классическому рассмотрению Бардина-Стефена (Bardeen-Stephen) [56], для сверхпроводников II рода магнитосопротивление пропорционально $\sin ^{2}(\angle \mathbf{H}, \mathbf{I})$, т. е. при $\mathbf{H} \perp \mathbf{I}$ (что соответствует условиям эксперимента) разрушение куперовских пар наиболее эффективное и магнитосопротивление максимально [48,49,57-61]. Для микроскопических токов $\mathbf{I}_{m}$ (см. рис. $1, b), R \sim \sin ^{2}\left(\angle \mathbf{H}, \mathbf{I}_{m}\right)$. С понижением температуры (например, от 80 до $4.2 \mathrm{~K}$ - см. рис. 5, $a$ и $6, a)$, величина намагниченности, определяющая индуцированное поле $B_{\text {ind }}$, возрастает более чем на порядок. Возможно, при сильном возрастании эффективного поля носителям будет предпочтительнее туннелировать через „соседнюю гранулу“, если при этом угол между $\mathbf{B}_{\text {ind }}$ и $\mathbf{I}_{m}$ мал. Фактически, микроскопические траектории тока могут „обтекать“ межгранульные границы, в которых $\mathbf{B}_{\text {eff }} \perp$ I. Схематически такое перераспределение микроскопических траекторий тока показано на рис. 1, c (в сравнении с рис. 1, $b$ ). Туннелирование в этом случае будет осуществляться через межгранульные промежутки, в которых $\angle \mathbf{B}_{\text {eff }}, \mathbf{I}_{m}<90^{\circ}$, а следовательно и $\angle \mathbf{H}, \mathbf{I}_{m}$ менее $90^{\circ}$. Тогда влияние внешнего поля будет меньше, и будет „работать“ проекция Н на плоскость $Z$, перпендикулярную траектории микроскопического тока (см. рис. 1,c). Величина этой проекции может быть записана как $H \cos \beta$, где $\beta=\angle \mathbf{I}, \mathbf{I}_{m}$, как показано на рис. $1, c$. На рис. $6, c$ приведена зависимость $B_{\text {eff }}(H)$ при $\alpha=20$, причем, в выражении (2) внешнее поле было взято с коэффициентом 0.5 , т. е. $\beta=60^{\circ}$. Конечно, нельзя сказать, что эта зависимость $B_{\text {eff }}(H)$ хорошо описывает поведение магнитосопротивления (рис. 6, $b$ ). Тем не менее, значения $B_{\mathrm{eff}}(H \approx 60 \mathrm{kOe})$ и $B_{\mathrm{eff}}\left(H_{\mathrm{inc}} \approx 1.5 \mathrm{kOe}\right)$ (поле $H_{\text {inc }} \approx 1.5 \mathrm{kOe}$ соответствует локальному максимуму зависимости $B_{\text {eff }}\left(H_{\text {inc }}\right)$ ) уже близки (в отличии от подобных значений для зависимости $B_{\text {eff }}(H)$ при $\alpha=30)$. И если принять во внимание, что при перераспределении траекторий тока параметр $\alpha$ может быть зависимым от внешнего поля (это достаточно сложно учесть в рамках простого выражения (2)), то можно сказать, что при замене „,H $\rightarrow H \cos \beta^{\text {“ }}$ зависимость $B_{\text {eff }}(H)$, хотя и качественно, но лучше объясняет гистерезис магнитосопротивления при $T=4.2 \mathrm{~K}$. Отметим, что полученное значение $\beta$ означает, что микроскопические токи могут отклоняться от направления макроскопического тока до $\sim 60^{\circ}$, что вполне ожидаемо в рамках данного сценария.

\section{4. Заключение}

Суммируем результаты, полученные в настоящей работе. На образце гранулярного ВТСП $\mathrm{YBa}_{2} \mathrm{Cu}_{3} \mathrm{O}_{7-\delta}$, обладающем достаточно высокими транспортными характеристиками $\left(J_{C}(T=77 \mathrm{~K}) \approx 150 \mathrm{~A} / \mathrm{cm}^{2}, J_{C}(T=4.2 \mathrm{~K}) \approx\right.$ $\approx 1.5 \mathrm{kA} / \mathrm{cm}^{2}$ при $\left.H=0\right)$, исследованы гистерезисные зависимости магнитосопротивления при различных плотностях транспортного тока как в диапазоне температур от $77 \mathrm{~K}$ до температуры перехода $T_{C}$, так и при $T=4.2 \mathrm{~K}$. Измерения включали в себя широкий диапазон транспортного тока и магнитных полей (до $90 \mathrm{kOe}$ ). Анализ полученных результатов проводился в рамках развиваемой авторами модели поведения гранулярного ВТСП во внешнем магнитном поле, в которой магнитосопротивление является функцией эффективного поля в межгранульной среде $-B_{\text {eff }}(H)=|H-4 \pi \alpha M(H)|$. 
В области высоких температур $\left(77 \mathrm{~K}-T_{C}\right)$ все особенности зависимости $R(H)$ адекватно описываются в рамках модели. К ним относятся: 1) независимость величины полевой ширины $\Delta H$ гистерезиса $R(H)$ от транспортного тока, 2) достаточно большая величина $\Delta H$, что характеризует сильное сжатие магнитного потока в межгранульной среде, 3) наличие выраженного локального максимума зависимости $R(H)$ при увеличении внешнего поля. Первые две особенности также характерны и для данных при $4.2 \mathrm{~K}$, однако локальный максимум, ярко проявляющийся в области высоких температур, на зависимости $R(H)$ отсутствует. Это свидетельствует о возникновении дополнительного фактора, влияющего на процессы диссипации в межгранульной среде в области низких температур. Этим фактором может быть перераспределение траекторий микроскопического тока, происходящее с изменением внешнего магнитного поля. Иными словами, в области низких температур, в условиях сильного сжатия потока возможно изменение микроскопических траекторий тока, когда предпочтительнее туннелирование через те межгранульные границы, в которых направление микротока будет не перпендикулярно линиям напряженности эффективного поля (при условиях, когда $\mathbf{H} \perp \mathbf{I}$ ). Исследуемый в работе образец обладает характеристиками, типичными для гранулярных ВТСП-материалов иттриевой системы, что дает основания обобщать полученные выводы, по крайней мере, на класс гранулярных материалов данной ВТСП-системы.

\section{Благодарности}

Авторы благодарят Д.М. Гохфельда за обсуждение результатов. Часть измерений транспортных свойств проводились установке PPMS-6000 Центра коллективного пользования ФИЦ КНЦ СО РАН.

\section{Конфликт интересов}

Авторы заявляют, что у них нет конфликта интересов.

\section{Список литературы}

[1] J. Jung, M.A.-K. Mohamed, S.C. Cheng, J.P. Franck. Phys. Rev. B 42, 10, 6181 (1990).

[2] B. Andrzejewski, E. Guilmeau, Ch. Simon. Supercond. Sci. Technol. 14, 904 (2001).

[3] Э.Б. Сонин. Письма в ЖЭТФ 47, 415 (1988).

[4] D.-X. Chen, R.W. Cross, A. Sanchez. Cryogenics 33, 695 (1993).

[5] В.В. Вальков, Б.П. Хрусталев. ЖЭТФ 107, 1221 (1995).

[6] Д.М. Гохфельд. ФТТ 56, 12, 2298 (2014).

[7] M.A. Dubson, S.T. Herbet, J.J. Calabrese, D.C. Harris, B.R. Patton, J.C. Garland. Phys. Rev. Lett. 60, 1061 (1988).

[8] M.I. Petrov, S.N. Krivomazov, B.P. Khrustalev, K.S. Aleksandrov. Solid State Commun. 82, 453 (1992).

[9] M.I. Petrov, D.A. Balaev, B.P. Khrustalev, K.S. Aleksandrov. Physica C 235-240, 3043 (1994).
[10] J.E. Evetts, B.A. Glowacki. Cryogenics 28, 641 (1988).

[11] E. Altshuler, J. Musa, J. Barroso, A.R.R. Papa, V. Venegas. Cryogenics 33, 308 (1993).

[12] P. Mune, E. Govea-Alcaide, R.F. Jardim. Physica C 354, 275 (2001).

[13] P. Mune, F.C. Fonseca, R. Muccillo, R.F. Jardim. Physica C 390, 363 (2003).

[14] D.A. Balaev, D.M. Gokhfeld, S.I. Popkov, K.A. Shaykhutdinov, M.I. Petrov. Physica C 460-462, 1307 (2007).

[15] D.A. Balaev, A.A. Dubrovskii, S.I. Popkov, D.M. Gokhfeld, S.V. Semenov, K.A. Shaykhutdinov, M.I. Petrov. Phys. Solid State 54, 11, 2155 (2012).

[16] C.A.M. dos Santos, M.S. da Luz, B. Ferreira, A.J.S. Machado. Physica C 391, 345 (2003).

[17] S. Shifang, Z. Yong, P. Guoqian, Y. Daoq, Z. An, C. Zuyao, Q. Yitai, K. Eiyan, Z. Qirui. Europhys. Lett. 6, 4, 359 (1988).

[18] L. Ji, M.S. Rzchowski, N. Anand, M. Tinkham. Phys. Rev B 47, 470 (1993).

[19] M. Prester, E. Babic, M. Stubicar, P. Nozar'. Phys. Rev. B 49, 10, 6967 (1994).

[20] M. Prester. Supercond. Sci. Technol. 11, 333 (1998).

[21] D. Daghero, P. Mazzetti, A. Stepanescu, P. Tura. Phys. Rev. B 66, 13, 11478 (2002).

[22] Н.Д. Кузьмичев. Письма в ЖЭТФ 74, 291 (2001).

[23] Н.Д. Кузьмичев. ФТТ 43, 1934 (2001).

[24] А.А. Суханов, В.И. Омельченко. ФНТ 29, 4, 396 (2003).

[25] В.В. Деревянко, Т.В. Сухарева, В.А. Финкель. ФТТ 46, 10 , 1740 (2004).

[26] В.В. Деревянко, Т.В. Сухарева, В.А. Финкель. ФТТ 49, 10, 1744 (2007).

[27] Т.В. Сухарева, В.А. Финкель. ФТТ 50, 6, 961 (2008).

[28] В.В. Деревянко, Т.В. Сухарева, В.А. Финкель. ЖТФ 78, 36 (2008).

[29] Т.В. Сухарева, В.А. Финкель. ФТТ 53, 5, 858 (2011).

[30] В.В. Деревянко, Т.В. Сухарева, В.А. Финкель, Ю.Н. Шахов. ФTT 56, 625 (2014).

[31] В.В. Деревянко, Т.В. Сухарева, В.А. Финкель. ФТТ 59, 8, 1470 (2017).

[32] В.В. Деревянко, Т.В. Сухарева, В.А. Финкель. ФТТ 60, 3, 465 (2018).

[33] М.А. Васютин. Письма в ЖТФ 39, 9 (2013).

[34] Д.А. Балаев, Д.М. Гохфельд, А.А. Дубровский, С.И. Попков, К.А. Шайхутдинов, М.И. Петров. ЖЭТФ 132, 1340 (2007).

[35] Д.А. Балаев, А.А. Дубровский, К.А. Шайхутдинов, С.И. Попков, Д.М. Гохфельд, Ю.С. Гохфельд, М.И. Петров. ЖЭТФ 135, 271 (2009).

[36] Д.А. Балаев, А.А. Дубровский, С.И. Попков, К.А. Шайхутдинов, М.И. Петров. ФТТ 50, 972 (2008).

[37] К.А. Шайхутдинов, Д.А. Балаев, С.И. Попков, М.И. Петров. ФТТ 51, 1046 (2009).

[38] Д.А. Балаев, А.А. Быков, С.В. Семенов, С.И. Попков, А.А. Дубровский, К.А. Шайхутдинов, М.И. Петров. ФТТ 53, 5, 865 (2011).

[39] D.A. Balaev, S.I. Popkov, S.V. Semenov, A.A. Bykov, E.I. Sabitova, A.A. Dubrovskiy, K.A. Shaikhutdinov, M.I. Petrov. J. Supercond. Nov. Magn. 24, 2129 (2011).

[40] Д.А. Балаев, С.И. Попков, К.А. Шайхутдинов, М.И. Петров, Д.М. Гохфельд. ФТТ 56, 8, 1492 (2014).

[41] Д.М. Гохфельд, Д.А. Балаев, С.В. Семенов, М.И. Петров. ФТT 57, 11, 2090 (2015). 
[42] M. Olutas, A. Kilic, K. Kilic, A. Altinkok. J. Supercond. Nov. Magn. 26, 3369 (2013).

[43] A. Altinkok, K. Kilic, M. Olutas, A. Kilic. J. Supercond. Nov. Magn. 26, 3085 (2013).

[44] M. Olutas, A. Kilic, K. Kilic, A. Altinkok. Eur. Phys. J. B 85, 382 (2012).

[45] D.A. Balaev, S.I. Popkov, E.I. Sabitova, S.V. Semenov, K.A. Shaykhutdinov, A.V. Shabanov, M.I. Petrov. J. Appl. Phys. 110, 093918 (2011).

[46] D.A. Balaev, S.V. Semenov, M.I. Petrov. J. Supercond. Nov. Magn. 27, 1425 (2014).

[47] Д.А. Балаев, С.В. Семенов, М.И. Петров. ФТТ 55, 12, 2305 (2013).

[48] С.В. Семенов, Д.А. Балаев, М.А. Почекутов, Д.А. Великанов. ФТТ 59, 7, 1267 (2017).

[49] D.A. Balaev, S.V. Semenov, M.A. Pochekutov. J. Appl. Phys. 122, 123902 (2017).

[50] S.V. Semenov, D.A. Balaev. Physica C 550, 19 (2018).

[51] S.V. Semenov, D.A. Balaev. J. Supercond. Nov. Magn. 32, 2409 (2019).

[52] S.V. Semenov, A.D. Balaev, D.A. Balaev. J. Appl. Phys. 125, 033903 (2019).

[53] А.Д. Балаев, Ю.В. Бояршинов, М.М. Карпенко, Б.П. Хрусталев. ПТЭ 3, 167 (1985).

[54] Д.А. Балаев, А.А. Дубровский, С.И. Попков, Д.М. Гохфельд, С.В. Семенов, К.А. Шайхутдинов, М.И. Петров. ФТT 54, 11, 11 (2012).

[55] М.А. Васютин, Н.Д. Кузьмичев, Д.А. Шилкин. ФТТ 58, 2, 231 (2016).

[56] J. Barden, M.J. Stephen. Phys. Rev. 140, A1197 (1965).

[57] D. Lopez, F. de la Cruz F. Phys. Rev. B 43, 13, 11478 (1991).

[58] D. Lopez, R. Decca, F. de la Cruz. Supercond. Sci. Technol. 5, 5, 276 (1992).

[59] O.V. Gerashchenko, S.L. Ginzburg. Supercond. Sci. Technol. 13, 332 (2000).

[60] D.A. Balaev, A.G. Prus, K.A. Shaukhutdinov, D.M. Gokhfeld, M.I. Petrov. Supercond. Sci. Technol. 20, 495 (2007).

[61] A. Kilic, K. Kilic, S. Senoussi, K. Demir. Physica C 294, 203 (1998).

Редактор Ю.Э. Китаев 